\title{
Even low-risk individuals can benefit from statin therapy
}

A new meta-analysis of statin therapy indicates that the benefits of lowering LDL cholesterol in patients at high risk of major vascular events are maintained when low-risk individuals take these drugs. If this strategy proves cost-effective, current guidelines might need to be reviewed.

Statins are widely used to lower LDL-cholesterol levels and thereby reduce risk in patients with a history of cardiovascular disease. Over the past 20 years, an international consortium of clinicians and researchers, the Cholesterol Treatment Trialists' (CTT) Collaboration, has collected data on approximately 175,000 individuals enrolled in 27 trials of statin treatment. Previously, the CTT Collaboration showed that "lowering of LDL cholesterol by $1 \mathrm{mmol} / \mathrm{l}$ with a standard statin regimen reduced the incidence of major vascular events by around a fifth, and that further reductions in LDL cholesterol with more intensive statin regimens yielded further reductions in risk."

However, according to Professor Colin Baigent from the University of Oxford, UK, and a member of the writing committee of the CTT Collaboration, "around half of all heart attacks and strokes occur in people with no previous history of [vascular] disease, so the prevention of such events with statins requires the selection and

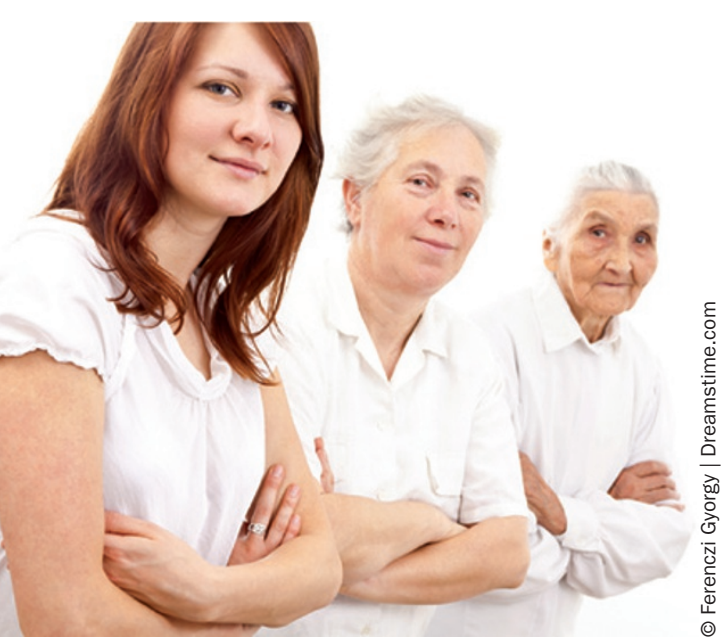

treatment of apparently healthy people." Primary prevention with statins in lowrisk individuals is controversial. "In a 2011 Cochrane Collaboration meta-analysis ... it was unclear whether the benefits of statin therapy outweighed the hazards in primary prevention," says Professor Baigent. "We were particularly concerned to clarify this area."

In their latest meta-analysis, the CTT Collaboration included individuals enrolled in 22 trials in which statin therapy was compared with a control $(n=134,537)$, and five trials in which high-dose and low-dose statin regimens were compared $(n=39,612)$. The individuals were then allocated to one of five groups (risk $<5 \%$, $\geq 5 \%$ to $<10 \%, \geq 10 \%$ to $<20 \%, \geq 20 \%$ to $<30 \%$, or $\geq 30 \%$ ) depending on their baseline 5-year risk of major vascular events (nonfatal myocardial infarction, coronary death, stroke, or coronary revascularization) while on control therapy (either no statin or low-dose statin). In each group, the rate ratio (RR) per $1.0 \mathrm{mmol} / \mathrm{l}$ of LDL-cholesterol reduction with statin therapy was estimated.

Across all the groups, lowering of LDLcholesterol levels reduced the risk of major vascular events (RR 0.79, 95\% CI 0.77-0.81, $P<0.0001)$. Importantly, the proportional reduction was similar in patients with a low baseline risk (RR 0.62, 95\% CI 0.47-0.81 for risk <5\%; RR 0.69, 95\% CI 0.60-0.79 for risk $\geq 5 \%$ to $<10 \%$ ) compared with those with a higher baseline risk (RR 0.79 , 95\% CI $0.74-0.85$ for risk $\geq 10 \%$ to $<20 \%$; RR $0.81,95 \%$ CI $0.77-0.86$ for risk $\geq 20 \%$ to $<30 \%$; RR 0.79 , 95\% CI 0.74-0.84 for risk $\geq 30 \%$ ).

The reduction in the risk of major vascular events in low-risk individuals was mainly attributable to reductions in major coronary events-nonfatal myocardial infarction or coronary revascularization. The reduction in stroke risk was similar in all five groups of patients, and did not differ when subdivided into ischemic or hemorrhagic stroke. Overall, vascular mortality was proportionally reduced by $12 \%$ per $1.0 \mathrm{mmol} / 1$ reduction in LDL cholesterol (RR 0.88, CI 0.84-0.91, $P<0.0001$ ).

In individuals with no history of vascular disease, the proportional reduction in the risk of major vascular events was similar in the two low-risk groups compared with the higher-risk groups. Primary prevention in these individuals reduced the risk of both vascular (RR 0.85, 95\% CI 0.77-0.95, $P=0.004$ ) and all-cause (RR 0.91, 95\% CI $0.85-0.97, P=0.007)$ mortality.

The meta-analysis showed no evidence of an increased risk of cancer incidence or mortality, or nonvascular causes of death. Statins have previously been associated with small increases in the risk of myopathy, rhabdomyolysis, incident diabetes mellitus, or (particularly in Asian populations) hemorrhagic stroke. However, the authors claim that no longterm adverse effects "are sufficiently large to outweigh the persistent benefits of statin therapy". Therefore, "statin therapy is beneficial even among those at the very lowest risk of major vascular events, and the benefits greatly exceed the known hazards," summarizes Professor Baigent.

The CTT Collaboration now plans to determine whether statin treatment in low-risk individuals is cost-effective. People with a 5-year risk of major vascular events $<10 \%$ are not normally considered for statin therapy, and Professor Baigent and colleagues propose that "the current guidelines for offering statin therapy to apparently healthy people may need to be reviewed. In the UK, the National Institute for Health and Clinical Excellence has indicated that it will be reviewing this research as part of its current review that is scheduled to report in 2013."

Gregory B. Lim
Original article Cholesterol Treatment Trialists' (CTT) Collaborators. The effects of lowering LDL cholesterol with statin therapy in people at low risk of vascular disease: meta-analysis of individual data from 27 trials. Lancet doi:10.1016/S0140-6736(12)60367-5 